Journal of Nonlinear Mathematical Physics

\title{
Bilinear identities for the constrained modified KP hierarchy
}

Huizhan Chen, Lumin Geng, Na Li, Jipeng Cheng

To cite this article: Huizhan Chen, Lumin Geng, Na Li, Jipeng Cheng (2019) Bilinear identities for the constrained modified KP hierarchy, Journal of Nonlinear Mathematical Physics 26:2, 240-254, DOI: https://doi.org/10.1080/14029251.2019.1591721

To link to this article: https://doi.org/10.1080/14029251.2019.1591721

Published online: 04 January 2021 


\title{
Bilinear identities for the constrained modified KP hierarchy
}

\author{
Huizhan Chen, Lumin Geng, $\mathrm{Na} \mathrm{Li}$ and Jipeng Cheng* \\ School of Mathematics, China University of Mining and Technology, \\ Xuzhou, Jiangsu 221116, P. R. China \\ chengjp@cumt.edu.cn
}

Received 19 September 2018

Accepted 29 November 2018

\begin{abstract}
In this paper, we mainly investigate an equivalent form of the constrained modified KP hierarchy: the bilinear identities. By introducing two auxiliary functions $\rho$ and $\sigma$, the corresponding identities are written into the Hirota forms. Also, we give the explicit solution forms of $\rho$ and $\sigma$.
\end{abstract}

Keywords: the constrained mKP hierarchy; the bilinear identities; tau functions.

2010 MSC: 35Q53, 37K10, 37K40

\section{Introduction}

Recently, people have paid much attention to the modified Kadomtsev-Petviashvili (mKP) hierarchy $[10,12]$ in the field of integrable systems, such as the gauge transformations [2,21], additional symmtries [3], the squared eigenfunction symmetries [3, 19,20] and the tau functions [3,25]. There are many versions $[4,6,11,13-15,21]$ of the $\mathrm{mKP}$ hierarchy, which are all trying to generalize the Miura link [22] between KdV and $\mathrm{mKdV}$ to the KP case. In this paper, we will only consider the Kupershmidt-Kiso version [13-15, 21]. By considering different reduction conditions on the Lax operator $L$, one can obtain the different kinds of sub-hierarchies. One of the most important subhierarchies is called the constained mKP (cmKP) hierarchy (see (2.16) in Section 2) [3,20], which is a generalization of the reduction procedure from the $\mathrm{mKP}$ hierarchy to the generalized $\mathrm{mKdV}$ hierarchy. In this paper, we will discuss the bilinear formulations of the cmKP hierarchy.

The bilinear identity $[7,10]$ is an important equivalent formulation of the integrable hierarchies. From the bilinear identity, one can easily obtain the Hirota equations $[7,9,10]$. And also it is very helpful in the discussion of the tau functions $[7,10]$. There is much research on the bilinear identity of different integrable hierarchies, for example, the constrained KP and BKP hierarchies [5, 17, $23,24]$, the extended bigraded Toda hierarchy $[16,18]$, the integrable hierarchies with with selfconsistent sources $[8,26]$. In this paper, we firstly derive three bilinear identities, from the evolution equations of the (adjoint) wave functions and the constraint on the Lax operator. Then we show that these three bilinear identities can fully characterize the cmKP hierarchy. By introducing two auxiliary functions $\rho$ and $\sigma$, the bilinear identities are written into the Hirota forms. At last, we give some solutions for $\rho$ and $\sigma$.

This paper is organized in the following way. In Section 2, some basic facts about the mKP hierarchy are introduced. The bilinear identities for the cmKP hierarchy are derived in Section 3.

${ }^{*}$ Corresponding author. 
Section 4 is devoted to the Hirota's bilinear equations of the corresponding tau-functions and the explicit forms of $\rho$ and $\sigma$. At last, some conclusions and discussions are given in Section 5.

\section{Reviews of the mKP hierarchy}

The definition of the mKP hierarchy is based on the theory of pseudo-differential operators, so we firstly introduce the knowledge of pseudo-differential operators $[7,14,21]$. The algebraic multiplication of $\partial^{i}$ with the multiplication operator $f$ is given by the usual Leibnitz rule

$$
\partial^{i} f=\sum_{j \geq 0}\left(\begin{array}{l}
i \\
j
\end{array}\right) f^{(j)} \partial^{i-j}, \quad i \in \mathbb{Z},
$$

where $f^{(j)}=\frac{\partial^{j} f}{\partial x^{j}}$. For $A=\sum_{i} a_{i} \partial^{i}, A_{\geq k}=\sum_{i \geq k} a_{i} \partial^{i}, A_{<k}=\sum_{i<k} a_{i} \partial^{i}$ and $A_{[k]}=a_{k}$. In this paper, for any pseudo- differential operator $A$ and a function $f$, the symbol $A(f)$ will indicate the action of $A$ on $f$, whereas the symbol $A f$ or $A \cdot f$ will denote the operator product of $A$ and $f$, and $*$ stands for the conjugate operation: $(A B)^{*}=B^{*} A^{*}, \partial^{*}=-\partial, f^{*}=f$.

The mKP hierarchy in Kupershmidt-Kiso version $[2,13-15,21]$ is defined as the following Lax equation

$$
L_{t_{n}}=\left[\left(L^{n}\right)_{\geq 1}, L\right], n=1,2,3, \ldots
$$

with the Lax operator $L$ given below

$$
L=\partial+u_{0}+u_{1} \partial^{-1}+u_{2} \partial^{-2}+u_{3} \partial^{-3}+\cdots .
$$

Here $\partial=\partial_{x}$ and $u_{i}=u_{i}\left(t_{1}=x, t_{2}, \ldots\right)$. The Lax operator $L$ of the mKP hierarchy can be expressed in terms of the dressing operator $Z$,

$$
L=Z \partial Z^{-1}
$$

where $Z$ is given by

$$
Z=z_{0}+z_{1} \partial^{-1}+z_{2} \partial^{-2}+\cdots\left(z_{0}^{-1} \text { exists }\right) .
$$

Then the Lax equation (2.2) is equivalent to

$$
Z_{t_{n}}=-\left(L^{n}\right)_{\leq 0} Z=-\left(Z \partial^{n} Z^{-1}\right)_{\leq 0} Z
$$

Define the wave and the adjoint functions of the mKP hierarchy in the following way:

$$
\begin{aligned}
& w(t, \lambda)=Z\left(e^{\xi(t, \lambda)}\right)=\left(z_{0}+z_{1} \lambda^{-1}+z_{2} \lambda^{-2}+\cdots\right) e^{\xi(t, \lambda)} \\
& w^{*}(t, \lambda)=\left(Z^{-1} \partial^{-1}\right)^{*}\left(e^{-\xi(t, \lambda)}\right)=\left(z_{0}^{-1}+z_{1}^{*} \lambda^{-1}+z_{2}^{*} \lambda^{-2}+\cdots\right) \lambda^{-1} e^{-\xi(t, \lambda)},
\end{aligned}
$$

where

$$
\xi(t, \lambda)=x \lambda+t_{2} \lambda^{2}+t_{3} \lambda^{3}+\cdots
$$

Then $w(t, \lambda)$ and $w^{*}(t, \lambda)$ satisfy the bilinear identity [3,25] below

$$
\operatorname{res}_{\lambda} w\left(t^{\prime}, \lambda\right) w^{*}(t, \lambda)=1,
$$

which is equivalent to the mKP hierarchy. Here $\operatorname{res}_{\lambda} \sum_{i} a_{i} \lambda^{i}=a_{-1}$. 
The Lax equation (2.2) is compatible with the linear system

$$
L w(t, \lambda)=\lambda w(t, \lambda), \quad \partial_{t_{n}} w(t, \lambda)=\left(L^{n}\right)_{\geq 1}(w(t, \lambda))
$$

and

$$
\left(\partial L \partial^{-1}\right)^{*} w^{*}(t, \lambda)=\lambda w^{*}(t, \lambda), \quad \partial_{t_{n}} w^{*}(t, \lambda)=-\left(\partial^{-1}\left(L^{n}\right)_{\geq 1}^{*} \partial\right)\left(w^{*}(t, \lambda)\right)
$$

It is proved in $[3,25]$ that there exist two tau functions $\tau_{1}$ and $\tau_{0}$ for the mKP hierarchy in Kupershmidt-Kiso version such that

$$
\begin{aligned}
w(t, \lambda) & =\frac{\tau_{0}\left(t-\left[\lambda^{-1}\right]\right)}{\tau_{1}(t)} e^{\xi(t, \lambda)}, \\
w^{*}(t, \lambda) & =\frac{\tau_{1}\left(t+\left[\lambda^{-1}\right]\right)}{\tau_{0}(t)} \lambda^{-1} e^{-\xi(t, \lambda)} .
\end{aligned}
$$

where $[\lambda]=\left(\lambda, \frac{\lambda^{2}}{2}, \frac{\lambda^{3}}{3}, \ldots\right)$. Then the bilinear identity (2.10) can be written into [3]

$$
\tau_{1}\left(t^{\prime}\right) \tau_{0}(t)=\operatorname{res} \lambda\left(\lambda^{-1} \tau_{0}\left(t^{\prime}-\left[\lambda^{-1}\right]\right) \tau_{1}\left(t+\left[\lambda^{-1}\right]\right) e^{\xi\left(t^{\prime}-t, \lambda\right)}\right)
$$

The $k$-constrained mKP hierarchy $[3,20]$ is defined by imposing the following constraints on the Lax operator,

$$
L^{k}=\left(L^{k}\right)_{\geq 1}+q \partial^{-1} r \partial
$$

where $q$ and $r$ are the eigenfunction and the adjoint eigenfunction of the mKP hierarchy respectively, satisfying

$$
q_{t_{n}}=\left(L^{n}\right)_{\geq 1}(q), \quad r_{t_{n}}=-\left(\partial\left(L^{n}\right)_{\geq 1} \partial^{-1}\right)^{*}(r)
$$

That is to say, the $k$-constrained mKP hierarchy are the system of (2.2), (2.3), (2.16) and (2.17). Next we list the powers of the Lax operators and the flows for $k=1$ and $k=2$.

\section{Case $k=1$}

- Powers of the Lax operators $(k=1)$

$$
\begin{aligned}
& L=\partial+q r-q r_{x} \partial^{-1}+q r_{x x} \partial^{-2}-q r_{x x x} \partial^{-3}+\cdots \\
& L^{2}=\partial^{2}+2 q r \partial+\left(q_{x} r-q r_{x}+q^{2} r^{2}\right)+\left(q r_{x x}-q_{x} r_{x}-2 q^{2} r r_{x}\right) \partial^{-1} \\
& +\left(q_{x} r_{x x}+2 q^{2} r_{x}^{2}+q q_{x} r r_{x}-q r_{x x x}+2 q^{2} r r_{x x}\right) \partial^{-2}+\cdots \\
& L^{3}=\partial^{3}+3 q r \partial^{2}+\left(3 q_{x} r+3 q^{2} r^{2}\right) \partial+q_{x x} r+q r_{x x}+3 q q_{x} r^{2}-3 q^{2} r r_{x}+q^{3} r^{3}-q_{x} r_{x} \\
& +\left(3 q^{2} r r_{x x}+q_{x} r_{x x}-q r_{x x x}-q_{x x} r_{x}-3 q r q_{x} r_{x}-3 q^{3} r^{2} r_{x}+3 q^{2} r_{x}^{2}\right) \partial^{-1}+\cdots
\end{aligned}
$$


- Flows of the cmKP hierarchy $(k=1)$

$$
\begin{aligned}
& u_{0 t_{2}}=q_{x x} r+2 q r r_{x}-q r_{x x}+2 q^{2} r r_{x}, \\
& q_{t_{2}}=q_{x x}+2 q r q_{x}, \quad r_{t_{2}}=-r_{x x}+2 q r r_{x} ;
\end{aligned}
$$

and

$$
\begin{aligned}
& u_{0 t_{3}}=q_{x x x} r+q r_{x x x}+3 r^{2}\left(q q_{x x}+\left(q_{x}\right)^{2}+q^{3} r_{x}\right) \\
& +3 q^{2}\left(r^{3} q_{x}-\left(r_{x}\right)^{2}+\left(q_{x}\right)^{2}\right)-3 q q_{x} r r_{x}, \\
& q_{t_{3}}=q_{x x x}+3 q r q_{x x}+3\left(q_{x}\right)^{2} r+3(q r)^{2} q_{x}, \\
& r_{t_{3}}=r_{x x x}-3(q r)_{x} r_{x}+3\left(q_{x}\right)^{2}+3(q r)^{2} r_{x} \text {. }
\end{aligned}
$$

\section{Case $k=2$}

- Powers of the Lax operators $(k=2)$

$$
\begin{aligned}
L= & \partial+u_{0}+\frac{1}{2}\left(q r-u_{0}^{2}-u_{0 x}\right) \partial^{-1}+\frac{1}{4}\left(-3 q r_{x}-q_{x} r\right. \\
& \left.+4 u_{0} u_{0 x}+u_{0 x x}-2 u_{0} q r+2 u_{0}^{3}\right) \partial^{-2}+\cdots \\
L^{2}= & \partial^{2}+2 u_{0} \partial+q r-q r_{x} \partial^{-1}+q r_{x x} \partial^{-2}-q r_{x x x} \partial^{-3}+\cdots \\
L^{3}= & \partial^{3}+3 u_{0} \partial^{2}+\frac{3}{2}\left(u_{0 x}+u_{0}^{2}+q r\right) \partial+\frac{1}{4}\left(u_{0 x x}+3 q_{x} r-3 q r_{x}+6 q r u_{0}-2 u_{0}^{3}\right) \\
& +\left(-\frac{1}{4} u_{0 x x x}+\frac{3}{8} q^{2} r^{2}+\frac{1}{2} u_{0}^{2} u_{0 x}+\frac{5}{4} q r_{x x}+\frac{1}{4} q_{x x} r-\frac{3}{4} u_{0} u_{0 x x}-\frac{5}{4} u_{0} q r_{x}\right. \\
& \left.+\frac{1}{4} u_{0} q_{x} r-\frac{3}{4} u_{0}^{2} q r-u_{0 x} q r+\frac{1}{8}\left(u_{0 x}\right)^{2}+\frac{3}{8} u_{0}^{4}\right) \partial^{-1}+\cdots
\end{aligned}
$$

- Flows of the cmKP hierarchy $(k=2)$

$$
\begin{aligned}
& u_{0 t_{2}}=(q r)_{x}, \\
& q_{t_{2}}=q_{x x}+2 u_{0} q_{x}, \\
& r_{t_{2}}=-r_{x x}+2 u_{0} r_{x} ;
\end{aligned}
$$

and

$$
\begin{aligned}
u_{0 t_{3}}= & \frac{3}{2}\left(q_{x x} r+q_{x} r_{x}+u_{0}(q r)_{x}\right)+\frac{3}{4}\left(u_{0 x}+u_{0}^{2}+q r\right)(1-q r) \\
& -u_{0}\left(\frac{3}{2} u_{0 x x}+4 u_{0} u_{0 x}-u_{0 x}\right), \\
q_{t_{3}}= & q_{x x x}+3 u_{0} q_{x x}+\frac{3}{2}\left(u_{0 x}+u_{0}^{2}+q r\right) q_{x}, \\
r_{t_{3}}= & r_{x x x}-3 u_{0} r_{x x}+\frac{3}{2}\left(-u_{0 x}+u_{0}^{2}+q r\right) r_{x} .
\end{aligned}
$$




\section{Bilinear identities}

In this section, we would like to discuss the bilinear identity formulation of the $k$-constrained mKP hierarchy. From the spectrum equation of the linear system (2.11), it is obvious that

$$
L^{k}(w(t, \lambda))=\left(\left(L^{k}\right)_{\geq 1}+q \partial^{-1} r \partial\right)(w(t, \lambda))=\lambda^{k} w(t, \lambda),
$$

that is

$$
\left(L^{k}\right)_{\geq 1}(w(t, \lambda))+q S(t, \lambda)=\lambda^{k} w(t, \lambda)
$$

where

$$
S_{x}(t, \lambda)=r w_{x}(t, \lambda)
$$

Similarly, from (2.12), we have

$$
\partial^{-1}\left(L^{k}\right)_{\geq 1}^{*} \partial\left(w^{*}(t, \lambda)\right)+r \hat{S}(t, \lambda)=\lambda^{k} w^{*}(t, \lambda),
$$

where

$$
\hat{S}_{x}(t, \lambda)=q w_{x}^{*}(t, \lambda)
$$

Before further dicussion, the following lemmas are needed.

Lemma 3.1 ([7]). Let $P$ and $Q$ be two pseudo-differential operators, then

$$
\operatorname{res}_{\lambda}\left[\left(P e^{x \lambda}\right)\left(Q e^{-x \lambda}\right)\right]=\operatorname{res}_{\partial} P Q^{*}
$$

where $Q^{*}$ is the formal adjoint of $Q$.

Lemma 3.2 ([7]). If $f(z)=\sum_{i=0}^{\infty} a_{i} z^{-i}$ is a standard series, one has the following operator identities:

$$
\operatorname{res}_{z}\left(\left(\sum_{n=-\infty}^{\infty} a_{n}(\zeta) z^{-n}\right) \frac{1}{1-z / \zeta}\right)=\left.\zeta\left(\sum_{n=1}^{\infty} a_{n}(\zeta) z^{-n}\right)\right|_{z=\zeta}
$$

Proposition 3.1. For the $k$-constrained $m K P$ hierarchy (2.16), the eigenfunctions $r(t)$ and $q(t)$ satisfy the following residue formulas:

$$
\begin{aligned}
& q(t) r\left(t^{\prime}\right)=\operatorname{res}_{\lambda} \lambda^{k} w(t, \lambda) w^{*}\left(t^{\prime}, \lambda\right) \\
& q(t)=\operatorname{res}_{\lambda} w(t, \lambda) \hat{S}\left(t^{\prime}, \lambda\right) \\
& r\left(t^{\prime}\right)=\operatorname{res}_{\lambda} S(t, \lambda) w^{*}\left(t^{\prime}, \lambda\right)
\end{aligned}
$$

with $t$ and $t$ ' being arbitrary and independent of each other. 
Proof. We first calculate the residue of $L^{k} \partial^{m}$ by Lemma 3.1 for an integer $m \geq-1$

$$
\begin{aligned}
(-1)^{m+1} q \partial_{x}^{m+1}(r) & =\operatorname{res}_{\partial}\left(L^{k} \partial^{m}\right) \\
& =\operatorname{res}_{\partial}\left(Z \partial^{k} Z^{-1} \partial^{m}\right) \\
& =\operatorname{res}_{\lambda}\left(Z \partial^{k}\left(e^{\xi(t, \lambda)}\right) \cdot(-\partial)^{m}\left(Z^{*}\right)^{-1}\left(e^{-\xi(t, \lambda)}\right)\right) \\
& =(-1)^{m+1} \operatorname{res}_{\lambda}\left(\lambda^{k} w(t, \lambda) \cdot \partial_{x}^{m+1}\left(w^{*}(t, \lambda)\right)\right) .
\end{aligned}
$$

Then by using the Taylor expansion

$$
f\left(t^{\prime}\right)=\sum\left(t_{1}^{\prime}-t_{1}\right)^{i_{1}} \cdots\left(t_{m}^{\prime}-t_{m}\right)^{i_{m}} \partial_{1}^{i_{1}} \cdots \partial_{m}^{i_{m}} f(t) / i_{1} ! \cdots i_{m} !
$$

and also using (2.12) and (2.17), one can obtain

$$
q(t) r\left(t^{\prime}\right)=\operatorname{res}_{\lambda} \lambda^{k} w(t, \lambda) w^{*}\left(t^{\prime}, \lambda\right)
$$

Further from (2.10), we find that the formula (3.13) can be written as follows:

$$
\begin{aligned}
q(t) r\left(t^{\prime}\right) & =\operatorname{res}_{\lambda} \lambda^{k} w(t, \lambda) w^{*}\left(t^{\prime}, \lambda\right) \\
& =\operatorname{res}_{\lambda} w(t, \lambda) \cdot\left(\partial_{x^{\prime}}\left(L^{k}\right)_{\leq 0} \partial_{x^{\prime}}^{-1}\right)^{*}\left(w^{*}\left(t^{\prime}, \lambda\right)\right) \\
& =\operatorname{res}_{\lambda} w(t, \lambda) \cdot r\left(t^{\prime}\right) \partial_{x^{\prime}}^{-1} q\left(t^{\prime}\right) \partial_{x^{\prime}}\left(w^{*}\left(t^{\prime}, \lambda\right)\right) \\
& =\operatorname{res}_{\lambda} w(t, \lambda) r\left(t^{\prime}\right) \hat{S}\left(t^{\prime}, \lambda\right) .
\end{aligned}
$$

By eliminating $r\left(t^{\prime}\right)$ on both sides of the upper form, we get (3.9). By the similar way, formula (3.13) can also be written the following form:

$$
\begin{aligned}
q(t) r\left(t^{\prime}\right) & =\operatorname{res}_{\lambda} \lambda^{k} w(t, \lambda) w^{*}\left(t^{\prime}, \lambda\right)=\operatorname{res}_{\lambda} L^{k} w(t, \lambda) w^{*}\left(t^{\prime}, \lambda\right) \\
& =\operatorname{res}_{\lambda}\left(L^{k}\right)_{\leq 0} w(t, \lambda) w^{*}\left(t^{\prime}, \lambda\right)=\operatorname{res}_{\lambda} q(t) S(t, \lambda) w^{*}\left(t^{\prime}, \lambda\right) .
\end{aligned}
$$

By eliminating $q(t)$ from both sides of the equation above, (3.10) can be proved.

Proposition 3.2. Conversely, let $w(t, \lambda)$ be a formal power series of the form $w(t, \lambda)=$ $\sum_{i=0}^{\infty} z_{i} \lambda^{-i} e^{\xi(t, \lambda)}, w^{*}(t, \lambda)=\left(z_{0}^{-1}+\sum_{i=1}^{\infty} z_{i}^{*} \lambda^{-i}\right) e^{\xi(t, \lambda)} \lambda^{-1}$, where $z_{i}$ and $z_{i}^{*}$ are functions of variable $t_{i}$. Both $w(t, \lambda)$ and $w^{*}(t, \lambda)$ satisfy (3.8)-(3.10). Then letting $Z=\sum_{i=0}^{\infty} z_{i} \partial^{-i}$, one has the following conclusions:

(i) $w(t, \lambda)=Z\left(e^{\xi(t, \lambda)}\right), \quad w^{*}(t, \lambda)=\left(Z^{-1} \partial^{-1}\right)^{*}\left(e^{-\xi(t, \lambda)}\right)$,

(ii) $\partial_{t_{n}} Z=\left(Z \partial^{n} Z^{-1}\right)_{\leq 0} Z$,

(iii) $\left(L^{k}\right)_{\leq 0}=q \partial^{-1} r \partial$. 
Proof. (i)-(ii) It is obvious that $w(t, \lambda)=Z\left(e^{\xi(t, \lambda)}\right)$. Let

$$
w^{*}(t, \lambda)=-\widetilde{Z} \partial^{-1}\left(e^{-\xi(t, \lambda)}\right)
$$

where $\widetilde{Z}=z_{0}^{-1}+\sum_{i=1}^{\infty} z_{i}^{*} \partial^{-1}$. By differentiating both sides of (3.9) with respect to $x^{\prime}$ and letting $t \leftrightarrow t^{\prime}$

$$
0=\operatorname{res}_{\lambda} w\left(t^{\prime}, \lambda\right) w^{*}(t, \lambda)_{x}
$$

Then

$$
0=\operatorname{res}_{\lambda}\left(z_{0}^{-1}\left(t^{\prime}\right) Z\right)\left(e^{\xi\left(t^{\prime}, \lambda\right)}\right) \cdot\left(z_{0}(t) \partial \widetilde{Z} \partial^{-1}\right)\left(e^{-\xi(t, \lambda)}\right) .
$$

Note that the highest order terms of $z_{0}^{-1}\left(t^{\prime}\right) Z_{1}$ and $z_{0}(t) \partial \widetilde{Z} \partial^{-1}$ in (3.18) are 1 , which implys the residue formula (3.18) can be viewed as the bilinear relation of the KP hierarchy. Thus we have

$$
z_{0}^{-1} Z \cdot \partial^{-1} \widetilde{Z}^{*} \partial z_{0}=1
$$

and $\phi \triangleq z_{0}^{-1} Z$ is the dressing operator of KP hierarchy, i.e. $\phi_{t_{n}}=-\left(\phi \partial^{n} \phi^{-1}\right)_{<0} \phi$. Therefore,

$$
w^{*}(t, \lambda)=-\widetilde{Z} \partial^{-1}\left(e^{-\xi(t, \lambda)}\right)=\left(Z^{-1} \partial^{-1}\right)^{*}\left(e^{-\xi(t, \lambda)}\right) .
$$

If set $\psi(t, \lambda)=\phi\left(e^{\xi(t, \lambda)}\right)$, then $\psi(t, \lambda)_{t_{n}}=B_{n}(\psi)$. Therefore

$$
\begin{aligned}
w(t, \lambda)_{t_{n}} & =\left(z_{0} \psi(t, \lambda)\right)_{t_{n}} \\
& =z_{0 t_{n}} \cdot\left(z_{0}^{-1} Z\right)\left(e^{\xi(t, \lambda)}\right)+z_{0} \cdot\left(B_{n} z_{0}^{-1} Z\right)\left(e^{\xi(t, \lambda)}\right) \\
& =\left(z_{0 t_{n}} \cdot z_{0}^{-1}+z_{0} \cdot B_{n} z_{0}^{-1}\right)(w(t, \lambda)) .
\end{aligned}
$$

It can be found from equation (3.21) that the derivatives of $w(t, \lambda)$ with respect to $t_{n}$ can be transformed into the action of the differential operators $\sum_{i \geq 0} a_{i} \partial^{i}$ on it. Then by Lemma 3.1

$$
\begin{aligned}
\operatorname{res}_{\lambda} \partial^{i} w(t, \lambda) w^{*}(t, \lambda) & =\operatorname{res}_{\lambda} \partial^{i} Z\left(e^{\xi(t, \lambda)}\right)\left(Z^{-1} \partial^{-1}\right)^{*}\left(e^{-\xi(t, \lambda)}\right) \\
& =\operatorname{res}_{\partial} \partial^{i} Z \cdot Z^{-1} \partial^{-1}=\operatorname{res}_{\partial} \partial^{i-1}=\delta_{i 0} .
\end{aligned}
$$

Taking into account (3.12), one can obtain

$$
\operatorname{res}_{\lambda} w(t, \lambda) w^{*}\left(t^{\prime}, \lambda\right)_{x^{\prime}}=1
$$

which is the bilinear identity of the mKP hierarchy, thus $\partial_{t_{n}} Z=\left(Z \partial^{n} Z^{-1}\right)_{\leq 0} Z$. 
(iii) Differentiating (3.8) with respect to $t_{n}^{\prime}$, one sees that

$$
\begin{aligned}
q(t) \partial_{n^{\prime}} r\left(t^{\prime}\right) & =-\operatorname{res}_{\lambda} \lambda^{k} w(t, \lambda)\left(\partial_{x^{\prime}}^{-1}\left(L^{n}\right)_{\geq 1}^{*} \partial_{x^{\prime}}\right)\left(w^{*}\left(t^{\prime}, \lambda\right)\right) \\
& =-\left(\partial_{x^{\prime}}^{-1}\left(L^{n}\right)_{\geq 1}^{*} \partial_{x^{\prime}}\right)\left(q(t) r\left(t^{\prime}\right)\right) \\
& =-q(t)\left(\partial_{x^{\prime}}^{-1}\left(L^{n}\right)_{\geq 1}^{*} \partial_{x^{\prime}}\right)\left(r\left(t^{\prime}\right)\right),
\end{aligned}
$$

which means that $r(t)$ is the adjoint eigenfunction. By the similar way, one show that $q(t)$ is the eigenfunction. Finally, by differentiating (3.8) with respect to $t_{n}^{\prime}$ and letting $t^{\prime}=t$,

$$
\begin{aligned}
q(t) \partial^{n}(r(t)) & =\operatorname{res}_{\lambda} \lambda^{k} w(t, \lambda) \cdot \partial^{n}\left(w^{*}\left(t^{\prime}, \lambda\right)\right) \\
& =\operatorname{res}_{\lambda}\left(L^{k} Z\right)\left(e^{\lambda x}\right) \cdot \partial^{n}\left(Z^{-1} \partial^{-1}\right)^{*}\left(e^{-\lambda x}\right) \\
& =(-1)^{n} \operatorname{res}_{\partial} L^{k} \partial^{n-1},
\end{aligned}
$$

that is,

$$
(-1)^{n} q(t) \partial^{n}(r(t))=\operatorname{res}_{\partial} L^{k} \partial^{n-1} .
$$

Formula (3.26) shows that the $\partial^{-n}$ term in $L^{k}$ is $(-1)^{n} q(t) r^{(n)}(t)$. Furthermore, $\sum_{n=1}^{\infty}(-1)^{n} q r^{(n)} \partial^{-n}=q \partial^{-1} r \partial$. This means that the nonpositive part of the Lax operator $L^{k}$ is the form (2.16). This completes the proof that (3.8), (3.9) and (3.10) fully characterize the $k$-constrained mKP hierarchy.

\section{Tau functions and Hirota bilinear equations}

In this section, we would like to rewrite the bilinear identities in Hirota form. Introduce two auxiliary functions $\rho(t)$ and $\sigma(t)$ such that

$$
q(t)=\frac{\rho(t)}{\tau_{1}(t)}, \quad r(t)=\frac{\sigma(t)}{\tau_{0}(t)},
$$

where $\tau_{0}(t)$ and $\tau_{1}(t)$ are defined by (2.13) and (2.14), then we have the explicit expressions for functions $S(t, \lambda)$ and $\hat{S}(t, \lambda)$.

\section{Proposition 4.1.}

$$
\begin{aligned}
& S(t, \lambda)=\frac{\sigma\left(t-\left[\lambda^{-1}\right]\right)}{\tau_{1}(t)} e^{\xi(t, \lambda)}, \\
& \hat{S}(t, \lambda)=\frac{\rho\left(t+\left[\lambda^{-1}\right]\right)}{\lambda \tau_{0}(t)} e^{-\xi(t, \lambda)} .
\end{aligned}
$$

Proof. In terms of the definition of $S(t, \lambda)$ (see (3.3)), the function $S$ can be expressed as

$$
S(t, \lambda)=K_{1}(t, \lambda) e^{\xi(t, \lambda)},
$$

where

$$
K_{1}(t, \lambda)=k_{0}+\frac{k_{1}}{\lambda}+\frac{k_{2}}{\lambda^{2}}+\cdots
$$


Due to

$$
\frac{1}{\left(1-z s_{1}\right)\left(1-z s_{2}\right)}=\left(\frac{1}{\left(1-z s_{2}\right)}-\frac{1}{\left(1-z s_{1}\right)}\right) \frac{1}{z\left(s_{2}-s_{1}\right)} .
$$

Define the operator $G(z) f(t)=f\left(t-\left[z^{-1}\right]\right)$. By setting the time variables $t_{n}^{\prime}=t_{n}-\frac{1}{n \xi_{1}^{n}}-\frac{1}{n \xi_{2}^{n}}$, and substituting (4.4) and (2.8) into (3.10), and using Lemma 3.2,

$$
\begin{aligned}
G\left(\xi_{1}\right) G\left(\xi_{2}\right) r(t) & =\operatorname{res}_{\lambda}\left(K_{1}(t, \lambda) e^{\xi(t, \lambda)} \hat{w}^{*}\left(t^{\prime}, \lambda\right) \lambda^{-1} e^{-\xi\left(t^{\prime}, \lambda\right)}\right) \\
& =\operatorname{res}_{\lambda}\left(\frac{K_{1}(t, \lambda) e^{\xi(t, \lambda)} G\left(\xi_{1}\right) G\left(\xi_{2}\right) \hat{w}^{*}(t, \lambda) \lambda-1 e^{-\xi(t, \lambda)}}{\left(1-\frac{\lambda}{\xi_{1}}\right)\left(1-\frac{\lambda}{\xi_{2}}\right)}\right) \\
& =\frac{\xi_{1}}{1-\xi_{1} / \xi_{2}}\left(K_{1}\left(t, \xi_{1}\right) G\left(\xi_{1}\right) G\left(\xi_{2}\right) \hat{w}^{*}\left(t, \xi_{1}\right) \frac{1}{\xi_{1}}-K_{1}\left(t, \xi_{2}\right) G\left(\xi_{1}\right) G\left(\xi_{2}\right) \hat{w}^{*}\left(t, \xi_{2}\right) \frac{1}{\xi_{2}}\right) .
\end{aligned}
$$

By setting $\xi_{1}=\lambda, \xi_{2} \rightarrow \infty$, the above equation reads

$$
\frac{G(\lambda) \sigma(t)}{G(\lambda) \tau_{0}(t)}=\frac{K_{1}(t, \lambda) \tau_{1}(t)}{G(\lambda) \tau_{0}(t)}
$$

We then get the expression of $S(t, \lambda)$ as follows

$$
S(t, \lambda)=K_{1}(t, \lambda) e^{\xi(t, \lambda)}=\frac{G(\lambda) \sigma(t)}{G(\lambda) \tau_{0}(t)} \cdot \frac{G(\lambda) \tau_{0}(t)}{\tau_{1}(t)}=\frac{\sigma\left(t-\left[\lambda^{-1}\right]\right)}{\tau_{1}(t)} e^{\xi(t, \lambda)}
$$

In a similar way, from the definition of $\hat{S}(t, \lambda)$, the function $\hat{S}$ can be expressed as

$$
\hat{S}(t, \lambda)=K_{2}(t, \lambda) e^{-\xi(t, \lambda)}=\left(\frac{\bar{k}_{1}}{\lambda}+\frac{\bar{k}_{2}}{\lambda^{2}}+\cdots\right) e^{-\xi(t, \lambda)} .
$$

By substituting (4.9) and (2.7) into the bilinear identity (3.9) and taking $t_{n}=t_{n}^{\prime}+\frac{1}{n z_{1}^{n}}+\frac{1}{n z_{2}^{n}}$,

$$
\begin{aligned}
& G\left(-z_{1}\right) G\left(-z_{2}\right) q\left(t^{\prime}\right) \\
& =\operatorname{res}_{\lambda}\left(G\left(-z_{1}\right) G\left(-z_{2}\right) \hat{w}\left(t^{\prime}, \lambda\right) e^{\xi\left(t^{\prime}, \lambda\right)+\xi\left(z_{1}, \lambda\right)+\xi\left(z_{2}, \lambda\right)} K_{2}\left(t^{\prime}, \lambda\right) e^{-\xi\left(t^{\prime}, \lambda\right)}\right) \\
& =\operatorname{res}_{\lambda}\left(\frac{K_{2}\left(t^{\prime}, \lambda\right) G\left(-z_{1}\right) G\left(-z_{2}\right) \hat{w}\left(t^{\prime}, \lambda\right)}{\left(1-\frac{\lambda}{z_{1}}\right)\left(1-\frac{\lambda}{z_{2}}\right)}\right) \\
& =\frac{z_{1}}{1-z_{1} / z_{2}}\left(K_{2}\left(t^{\prime}, z_{1}\right) G\left(-z_{1}\right) G\left(-z_{2}\right) \hat{w}\left(t^{\prime}, z_{1}\right)-K_{2}\left(t^{\prime}, z_{2}\right) G\left(-z_{1}\right) G\left(-z_{2}\right) \hat{w} w\left(t^{\prime}, z_{2}\right)\right) .
\end{aligned}
$$

By setting $z_{1}=\lambda, \quad z_{2}^{-1}=0$, we finally reach $\left(t \leftrightarrow t^{\prime}\right)$

$$
\frac{G(-\lambda) \rho(t)}{\lambda G(-\lambda) \tau_{1}(t)}=\frac{K_{2}(t, \lambda) \tau_{0}(t)}{G(-\lambda) \tau_{1}(t)}
$$

which immediately implies

$$
\hat{S}(t, \lambda)=\frac{\rho\left(t+\left[\lambda^{-1}\right]\right)}{\lambda \tau_{0}(t)} e^{-\xi(t, \lambda)}
$$


Proposition 4.2. The auxiliary functions $\sigma(t), \rho(t), \tau_{1}(t)$, and $\tau_{0}(t)$ satisfy the following bilinear equations:

$$
\begin{aligned}
& \rho(t) \sigma\left(t^{\prime}\right)=\operatorname{res}_{\lambda}\left(\lambda^{k-1} \tau_{0}\left(t-\left[\lambda^{-1}\right]\right) \tau_{1}\left(t^{\prime}+\left[\lambda^{-1}\right]\right) e^{\xi\left(t-t^{\prime}, \lambda\right)}\right) \\
& \rho(t) \tau_{0}\left(t^{\prime}\right)=\operatorname{res}_{\lambda}\left(\lambda^{-1} \tau_{0}\left(t-\left[\lambda^{-1}\right]\right) \rho\left(t^{\prime}+\left[\lambda^{-1}\right]\right) e^{\xi\left(t-t^{\prime}, \lambda\right)}\right) \\
& \sigma\left(t^{\prime}\right) \tau_{1}(t)=\operatorname{res}_{\lambda}\left(\lambda^{-1} \tau_{1}\left(t^{\prime}+\left[\lambda^{-1}\right]\right) \sigma\left(t-\left[\lambda^{-1}\right]\right) e^{\xi\left(t-t^{\prime}, \lambda\right)}\right)
\end{aligned}
$$

Proof. These equations can be proved by substituting (2.13), (2.14), (4.1)-(4.3) into (3.8)-(3.10).

Remark 4.1. From (4.13) and (4.14), one can find that $\left(\tau_{0}, \rho\right)$ and $\left(\sigma, \tau_{1}\right)$ can be viewed as the $\tau$ functions of the mKP hierarchy, since they share the same form with (2.15).

Next, we try to rewrite (4.12)-(4.14) into the Hirota forms. For a polynomial $P$, one can define the Hirota bilinear operator [9] as follows:

$$
\begin{aligned}
P(D) f(t) \cdot g(t) & =\left.P\left(\left(\frac{\partial}{\partial t_{1}}-\frac{\partial}{\partial t_{1}^{\prime}}\right),\left(\frac{\partial}{\partial t_{2}}-\frac{\partial}{\partial t_{2}^{\prime}}\right), \ldots\right)\left(f(t) g\left(t^{\prime}\right)\right)\right|_{t=t^{\prime}} \\
& =P\left(\partial_{y}\right)(f(t+y) g(t-y)) \mid y=0 .
\end{aligned}
$$

Here $P(D)=P\left(D_{1}, D_{2}, \ldots\right)$ and $\partial_{y}=\left(\partial_{y_{1}}, \partial_{y_{2}}, \ldots\right)$. Another important object is the Schur polynomials $p_{n}(t)$, which are defined in the following way,

$$
e^{\xi(t, \lambda)}=\sum_{n=0}^{\infty} p_{n}(t) \lambda^{n}
$$

One can find $p_{n}(t)$ owns the form below:

$$
p_{n}(t)=\sum_{\|\alpha\|=n} \frac{t^{\alpha}}{\alpha !}
$$

where $\alpha=\left(\alpha_{1}, \alpha_{2}, \ldots\right), \quad \alpha_{j} \geq 0, \quad\|\alpha\|=\sum_{j=0}^{\infty} j \alpha_{j}, \quad \alpha !=\alpha_{1} ! \alpha_{2} ! \cdots, \quad t^{\alpha}=t_{1}^{\alpha_{1}} t_{2}^{\alpha_{2}} \cdots$.

Proposition 4.3. The Hirota's bilinear forms of the cmKP hierarchy are

$$
\begin{aligned}
& \text { (1) } \sum_{\alpha+\beta=\gamma} \frac{(-2)^{\alpha}}{\alpha ! \beta !} p_{k+\|\alpha\|}(\widetilde{D}) D^{\beta} \tau_{1}(t) \cdot \tau_{0}(t)-\frac{1}{\gamma !} D^{\gamma} \sigma(t) \cdot \rho(t)=0, \\
& \text { (2) }\left(\sum_{\alpha+\beta=\gamma} \frac{(-2)^{\alpha}}{\alpha ! \beta !} p_{\|\alpha\|}(\widetilde{D}) D^{\beta}-\frac{(-1)^{|\gamma|}}{\gamma !} D^{\gamma}\right) \rho(t) \cdot \tau_{0}(t)=0, \\
& \text { (3) }\left(\sum_{\alpha+\beta=\gamma} \frac{(-2)^{\alpha}}{\alpha ! \beta !} p_{\|\alpha\|}(\widetilde{D}) D^{\beta}-\frac{(-1)^{|\gamma|}}{\gamma !} D^{\gamma}\right) \tau_{1}(t) \cdot \sigma(t)=0 .
\end{aligned}
$$

where $\widetilde{D}=\left(D_{1}, \frac{D_{2}}{2}, \frac{D_{3}}{3}, \ldots\right)$. 
Proof. (1) Changing the variables in (4.12), $t \longrightarrow t-y, t^{\prime} \longrightarrow t+y$,

$$
\begin{aligned}
\rho(t-y) \sigma(t+y) & =\operatorname{res}_{\lambda}\left(\lambda^{k-1} \tau_{0}\left(t-y-\left[\lambda^{-1}\right]\right) \tau_{1}\left(t+y+\left[\lambda^{-1}\right]\right) e^{\xi(-2 y, \lambda)}\right) \\
& =\operatorname{res}_{\lambda}\left(\lambda^{k-1} e^{\xi(-2 y, \lambda)} e^{\sum_{n=1}^{\infty}\left(\frac{1}{n \lambda^{n}}\right) \partial_{y_{n}}}\left(\tau_{0}(t-y) \tau_{1}(t+y)\right)\right) \\
& =\operatorname{res} \lambda\left(\sum_{j=0}^{\infty} p_{j}(-2 y) \lambda^{k+j-1} \sum_{n=0}^{\infty} p_{n}\left(\widetilde{\partial}_{y}\right) \lambda^{-n}\left(\tau_{0}(t-y) \tau_{1}(t+y)\right)\right) \\
& =\sum_{j=0}^{\infty} p_{j}(-2 y) p_{k+j}\left(\widetilde{\partial}_{y}\right)\left(\tau_{0}(t-y) \tau_{1}(t+y)\right) .
\end{aligned}
$$

By considering that one can expand $f(y)=f\left(y_{1}, y_{2}, \ldots\right)$ at $y=0$ in the following way

$$
f(y)=\left.\exp \left(\sum_{i=1}^{\infty} y_{i} \frac{\partial}{\partial z_{i}}\right) f(z)\right|_{z=0}
$$

one can further obtain

$$
\begin{aligned}
& \left.\exp \left(\sum_{i=1}^{\infty} y_{i} \frac{\partial}{\partial z_{i}}\right)(\rho(t-z) \sigma(t+z))\right|_{z=0} \\
& =\left.\exp \left(\sum_{i=1}^{\infty} y_{i} \frac{\partial}{\partial z_{i}}\right) \sum_{j=0}^{\infty} p_{j}(-2 y) \cdot p_{j}\left(\widetilde{\partial}_{z}\right)\left(\tau_{0}(t-z) \tau_{1}(t+z)\right)\right|_{z=0},
\end{aligned}
$$

where $\widetilde{\partial}_{z}=\left(\partial_{z_{1}}, \frac{1}{2} \partial_{z_{2}}, \frac{1}{3} \partial_{z_{3}}, \ldots\right)$. Therefore

$$
e^{\sum_{i=0}^{\infty} y_{i} D_{i}} \sigma(t) \cdot \rho(t)=\sum_{j=0}^{\infty} p_{j}(-2 y) p_{k+j}(\widetilde{D}) e^{\sum_{n=1}^{\infty} y_{n} D_{n}} \tau_{1}(t) \cdot \tau_{0}(t) .
$$

Next, one can rewrite (4.24) into a more explicit form. Before doing this, the next formula is needed.

$$
\exp \left(\sum_{j=0}^{\infty} y_{j}\right)=\sum_{|\alpha|=0}^{\infty} \frac{y^{\alpha}}{\alpha !}
$$

where $y=\left(y_{1}, y_{2}, \ldots\right), \quad|\alpha|=\sum_{k=1}^{\infty} \alpha_{k}$.

By (4.17) and (4.25), we can rewrite (4.24) as

$$
\begin{aligned}
\sum_{|\beta|=0}^{\infty} \frac{y^{\beta}}{\beta !} D^{\beta}(\sigma(t) \cdot \rho(t)) & =\sum_{j=0}^{\infty} \sum_{\|\alpha\|=j} \frac{(-2 y)^{\alpha}}{\alpha !} p_{k+j}(\widetilde{D}) \sum_{|\beta|=0} \frac{y^{\beta}}{\beta !} D^{\beta} \tau_{1}(t) \cdot \tau_{0}(t) \\
& =\sum_{\|\alpha\|=0,|\beta|=0} \frac{(-2 y)^{\alpha}}{\alpha !} p_{k+\|\alpha\|}(\widetilde{D}) \frac{y^{\beta}}{\beta !} D^{\beta} \tau_{1}(t) \cdot \tau_{0}(t) .
\end{aligned}
$$

Further,

$$
\sum_{\gamma}\left(\sum_{\alpha+\beta=\gamma} \frac{(-2)^{\alpha}}{\alpha ! \beta !} p_{k+\|\alpha\|}(\widetilde{D}) D^{\beta} \tau_{1}(t) \cdot \tau_{0}(t)-\frac{1}{\gamma !} D^{\gamma} \sigma(t) \cdot \rho(t)\right) y^{\gamma}=0
$$

Therefore, one can obtain (4.18). In the same way, it is easy to prove (4.19) and (4.20).

Equations in (4.18), (4.19) and (4.20) give rise to the hierarchy of Hirota bilinear equations corresponding to the $k$-constrained mKP hierarchy. Let us show some examples for $k=1$ and $k=2$. 
Case $k=1$

- For $\gamma=0$,

$$
\sigma \cdot \rho=D_{1} \tau_{1} \cdot \tau_{0}
$$

- For $\gamma=(1,0,0, \ldots)$,

$$
D_{1} \sigma \cdot \rho=-2 D_{2} \tau_{1} \cdot \tau_{0}
$$

- For $\gamma=(0,1,0, \ldots)$,

$$
\begin{aligned}
& D_{2} \sigma \cdot \rho+\left(D_{1} D_{2}+\frac{1}{3} D_{1}^{3}+2 D_{3}\right) \tau_{1} \cdot \tau_{0}=0 \\
& \left(D_{2}-D_{1}^{2}\right) \rho \cdot \tau_{0}=0, \quad\left(D_{2}+D_{1}^{2}\right) \sigma \cdot \tau_{1}=0 .
\end{aligned}
$$

- For $\gamma=(0,0,1, \ldots)$,

$$
\begin{aligned}
& D_{3} \sigma \cdot \rho+\left(2 D_{4}+D_{1} D_{3}+D_{2}^{2}+\frac{1}{12} D_{1}^{4}+D_{1}^{2} D_{2}\right) \tau_{1} \cdot \tau_{0}=0 \\
& \left(4 D_{3}-3 D_{1}^{3}-3 D_{1} D_{2}\right) \rho \cdot \tau_{0}=0 \\
& \left(4 D_{3}-3 D_{1}^{3}+3 D_{1} D_{2}\right) \tau_{1} \cdot \sigma=0 .
\end{aligned}
$$

\section{Case $k=2$}

- For $\gamma=0$,

$$
2 \sigma \cdot \rho=\left(D_{1}^{2}+D_{2}\right) \tau_{1} \cdot \tau_{0}
$$

- For $\gamma=(1,0,0, \ldots)$,

$$
D_{1} \sigma \cdot \rho+\left(\frac{1}{2} D_{1} D_{2}+\frac{2}{3} D_{3}+\frac{1}{2} D_{1}^{3}\right) \tau_{1} \cdot \tau_{0}=0
$$

- For $\gamma=(0,1,0, \ldots)$,

$$
\begin{aligned}
& D_{2} \sigma \cdot \rho+\left(\frac{1}{12} D_{1}^{4}+\frac{1}{4} D_{2}^{2}+\frac{2}{3} D_{1} D_{3}+\frac{1}{2} D_{4}\right) \tau_{1} \cdot \tau_{0}=0, \\
& \left(D_{2}-D_{1}^{2}\right) \rho \cdot \tau_{0}=0, \quad\left(D_{2}+D_{1}^{2}\right) \sigma \cdot \tau_{1}=0 .
\end{aligned}
$$

- For $\gamma=(0,0,1, \ldots)$,

$$
\begin{aligned}
& D_{3} \sigma \cdot \rho+\left(2 D_{5}+D_{2}^{2}+\frac{5}{2} D_{1} D_{4}+\frac{3}{2} D_{1}^{2} D_{3}+\frac{1}{2} D_{1}^{2} D_{2}\right. \\
& \left.+\frac{1}{4} D_{1} D_{2}^{2}+\frac{1}{2} D_{1}^{3} D_{2}+\frac{5}{12} D_{1}^{5}\right) \tau_{1} \cdot \tau_{0}=0, \\
& \left(4 D_{3}-3 D_{1}^{3}-3 D_{1} D_{2}\right) \rho \cdot \tau_{0}=0, \\
& \left(4 D_{3}-3 D_{1}^{3}+3 D_{1} D_{2}\right) \tau_{1} \cdot \sigma=0 .
\end{aligned}
$$


At last, we will use the gauge transformation to give the specific form of $\rho(t)$ and $\sigma(t)$. In [1], we have constructed the gauge transformation of the constrained mKP hierarchy. For the cmKP hierarchy $\left(L^{(j)}\right)_{\leq 0}^{k}=q^{(j)} \partial^{-1} r^{(j)} \partial$, using the $n$ steps of gauge transformation operator $T_{D}$,

$$
L^{(0)} \stackrel{T_{D}\left(q^{(0)}\right)}{\longrightarrow} L^{(1)} \stackrel{T_{D}\left(q^{(1)}\right)}{\longrightarrow} L^{(2)} \stackrel{T_{D}\left(q^{(2)}\right)}{\longrightarrow} \ldots \stackrel{T_{D}^{(n-1)}}{\longrightarrow} L^{(n)},
$$

we have

$$
\begin{aligned}
q^{(n)} & =(-1)^{n} \frac{W_{n+1}\left(q^{(0)}, \eta_{1}, \eta_{2}, \ldots, \eta_{n}\right)}{W_{n}\left(q^{(0)}, \eta_{1}, \eta_{2}, \ldots, \eta_{n-1}\right)} \\
r^{(n)} & =(-1)^{n-1} \frac{W_{n-1}\left(q^{(0)}, \eta_{1}, \eta_{2}, \ldots, \eta_{n-2}\right)}{W_{n}\left(q^{(0)}, \eta_{1}, \eta_{2}, \ldots, \eta_{n-1}\right)} \\
\tau_{0}^{(n)} & =\frac{W_{n}\left(q^{(0)}, \eta_{1}, \ldots, \eta_{n-1}\right)\left(\tau_{1}^{(0)}\right)^{n}}{\left(\tau_{0}^{(0)}\right)^{n-1}} \\
\tau_{1}^{(n)} & =\frac{W_{n+1}\left(q^{(0)}, \eta_{1}, \ldots, \eta_{n-1}, 1\right)\left(\tau_{1}^{(0)}\right)^{n+1}}{\left(\tau_{0}^{(0)}\right)^{n}}
\end{aligned}
$$

where

$$
T_{D}\left(q^{(j)}\right)=\left(\left(q^{(j)}\right)^{-1}\right)_{x}^{-1} \partial\left(q^{(j)}\right)^{-1}, \eta_{j}=\left(L^{(0)}\right)^{k j}\left(q^{(0)}\right) .
$$

Here we have used the results in [2] about the changes of the tau functions under the gauge transformations.

Similarly, we can construct another $n$-step gauge transformation using only $T_{I}$ :

$$
L^{(0)} \stackrel{T_{I}\left(r^{(0)}\right)}{\longrightarrow} L^{(1)} \stackrel{T_{I}\left(r^{(1)}\right)}{\longrightarrow} L^{(2)} \stackrel{T_{I}\left(r^{(2)}\right)}{\longrightarrow} \ldots \stackrel{T_{I}\left(r^{(n-1)}\right)}{\longrightarrow} L^{(n)},
$$

and

$$
\begin{aligned}
r^{(n)} & =\frac{W_{n+1}\left(r^{(0)}, \hat{\eta}_{1}, \hat{\eta}_{2}, \ldots, \hat{\eta}_{n}\right)}{W_{n+1}\left(1, r^{(0)}, \hat{\eta}_{1}, \hat{\eta}_{2}, \ldots, \hat{\eta}_{n-1}\right)}, \\
q^{(n)} & =\frac{W_{n}\left(1, r^{(0)}, \hat{\eta}_{1}, \hat{\eta}_{2}, \ldots, \hat{\eta}_{n-2}\right)}{W_{n}\left(r^{(0)}, \hat{\eta}_{1}, \hat{\eta}_{2}, \ldots, \hat{\eta}_{n-1}\right)}, \\
\tau_{0}^{(n)} & =\frac{W_{n+1}\left(1, r^{(0)}, \hat{\eta}_{1}, \ldots, \hat{\eta}_{n-1}\right)\left(\tau_{0}^{(0)}\right)^{n+1}}{\left(\tau_{1}^{(0)}\right)^{n}}, \\
\tau_{1}^{(n)} & =\frac{W_{n}\left(r^{(0)}, \hat{\eta}_{1}, \ldots, \hat{\eta}_{n-1}\right)\left(\tau_{0}^{(0)}\right)^{n}}{\left(\tau_{1}^{(0)}\right)^{n-1}},
\end{aligned}
$$

where

$$
T_{I}\left(r^{(j)}\right)=\left(r^{(j)}\right)^{-1} \partial^{-1}\left(r^{(j)}\right)_{x}, \hat{\eta}_{j}=\int\left(L^{(0) *}\right)^{k j}\left(r_{x}^{(0)}\right) d x .
$$

Also the transformed tau functions can be derived by using the results in [2].

Starting from the zero solution of the mKP hierarchy, i.e. $L^{(0)}=\partial, \tau_{0}^{(0)}=\tau_{1}^{(0)}=1$, we have the following results. 
Proposition 4.4. Under the gauge transformation operator $T_{D}(q)$,

$$
\begin{aligned}
& \rho^{(n)}(t)=(-1)^{n} \frac{W_{n+1}\left(q^{(0)}, \eta_{1}, \ldots \eta_{n}\right) W_{n+1}\left(q^{(0)}, \eta_{1}, \ldots \eta_{n-1}, 1\right)}{W_{n}\left(q^{(0)}, \eta_{1}, \ldots \eta_{n-1}\right)}, \\
& \sigma^{(n)}(t)=(-1)^{n-1} W_{n-1}\left(q^{(0)}, \eta_{1}, \ldots \eta_{n-2}\right) .
\end{aligned}
$$

Under the gauge transformation operator $T_{I}(r)$,

$$
\begin{aligned}
& \rho^{(n)}(t)=W_{n}\left(1, r^{(0)}, \hat{\eta}_{1}, \ldots \hat{\eta}_{n-2}\right) \\
& \sigma^{(n)}(t)=W_{n+1}\left(r^{(0)}, \hat{\eta}_{1}, \ldots \hat{\eta}_{n}\right) .
\end{aligned}
$$

\section{Conclusions and Discussions}

The main results of this paper are as follows. We firstly derive the bilinear identities of the constrained mKP hierarchy from the calculus of the pseudo-differential operators, which are summarized in Proposition 3.1 and Proposition 3.2. In order to write bilinear equations in the form of Hirota operators, we introduce the auxiliary functions $\rho$ and $\sigma$ in Section 4, and give their bilinear equations in Proposition 4.2. Then, the Hirota's bilinear forms of the cmKP hierarchy are given in Proposition 4.3. At last, we use the gauge transformation to give the specific form of $\rho(t)$ and $\sigma(t)$ in Proposition 4.4.

We have established the bilinear method to express the constrained mKP hierarchy, just from the constraint on the Lax operator and the evolution equations of the (adjoint) wave functions. Though there is the Miura link between the KP and mKP hierarchy [22], our results are still not obvious. Comparatively, it is more difficult to obtain the bilinear formulation, only by using the Miura link from the results in KP case. Another important point is the auxiliary functions $\rho$ and $\sigma$. Since $\left(\tau_{0}, \rho\right)$ and $\left(\sigma, \tau_{1}\right)$ can be viewed as the new tau functions of the mKP hierarchy, it will be very interesting to further understand $\rho$ and $\sigma$.

\section{Acknowledgments}

This work is supported by China Postdoctoral Science Foundation (Grant No. 2016M591949) and Jiangsu Postdoctoral Science Foundation (Grant No. 1601213C).

\section{References}

[1] H.Z. Chen, L.M. Geng, N. Li and J.P. Cheng, Solving the constrained modified KP hierarchy by gauge transformations, J. Nonlin. Math Phys., 26 (2019), 1-15.

[2] J.P. Cheng, The gauge transformation of modified KP hierarchy, J. Nonlin. Math. Phys., 25 (2018), 66-85.

[3] J.P. Cheng, M.H. Li and K.L. Tian, On the modified KP hierarchy: tau functions, squared, eigenfunction symmetries and additional symmetries, J. Geom. Phys., 134 (2018), 19-37.

[4] Y. Cheng, Modifying the KP, the $n$th constrained KP hierarchies and their Hamiltonian structures, Comm. Math. Phys., 171 (1995), 661-682.

[5] Y. Cheng and Y.J. Zhang, Bilinear equations for the constrained KP hierarchy, Inverse Problems, 10 (1994), L11-L17.

[6] L.A. Dickey, Modified KP and discrete KP, Lett. Math. Phys., 48 (1999), 277-289.

[7] L.A. Dickey, Soliton equations and Hamiltonian systems, second ed. (World scientific, Singapore, 2003). 
[8] A. Doliwa and R.L. Lin, Discrete KP equation with self-consistent sources, Phys. Lett. A, 378 (2014), 1925-1931.

[9] R. Hirota, The Direct method in soliton theory, (Cambridge University Press, 2004).

[10] M. Jimbo and T. Miwa, Solitons and infinite dimensional Lie algebras, Publ. RIMS, Kyoto Univ., 19 (1983), 943-1001.

[11] V. Kac and J. van de Leur, Equivalence of formulations of the mKP hierarchy and its polynomial taufunctions, Japan. J. Math., 13 (2018), 235-271.

[12] M. Kashiwara and T. Miwa, The $\tau$ function of the Kadomtsev-Petviashvili equation, Transfromation groups for soliton equations, I, Proc. Japan Acad. A, 57 (1981), 342-347.

[13] K. Kiso, A remark on the commuting flows defined by Lax equations, Prog. Theor. Phys., 83 (1990), $1108-1125$.

[14] B. Konopelchenko and W. Oevel, An $r$-matrix approach to nonstandard classes of integrable equations, Publ. RIMS Kyoto Univ., 29 (1993), 581-666.

[15] B. A. Kupershmidt, Mathematics of dispersive water waves, Comm. Math. Phys., 99 (1985), 51-73.

[16] C.Z. Li, J.S. He, Ke Wu and Yi Cheng, Tau function and Hirota bilinear equations for the extended bigraded Toda hierarchy, J. Math. Phys., 51 (2010), 043514.

[17] I. Loris and R. Willox, Bilinear form and solutions of the $k$-constrained Kadomtsev-Petviashvili hierarchy, Inverse Problems, 13 (1997), 411-420.

[18] T. Milanov, Hirota quadratic equations for the extended Toda hierarchy, Duke Math. J., 138 (2007), $161-178$

[19] W. Oevel and S. Carillo, Squared eigenfunction symmetries for soliton equations: Part I, J. Math. Anal. Appl., 217 (1998), 161-178.

[20] W. Oevel and S. Carillo, Squared eigenfunction symmetries for soliton equations: Part II, J. Math. Anal. Appl., 217 (1998), 179-199.

[21] W. Oevel and C. Rogers, Gauge transformations and reciprocal links in $2+1$ dimensions, Rev. Math. Phys., 5 (1993), 299-330.

[22] J.C. Shaw and M.H. Tu, Miura and auto-Backlund transformations for the $\mathrm{cKP}$ and $\mathrm{cmKP}$ hiearchies, J. Math. Phys., 38 (1997), 5756-5773.

[23] H.F. Shen, N.C. Lee and M.H. Tu, Operator approach to solutions of the constrained BKP hierarchy, J. Phys. A: Math. Theor, 44 (2011), 135205.

[24] H.F. Shen and M.H. Tu, On the constrained B-type Kadomtsev-Petviashvili hierarchy: Hirota bilinear equations and Virasoro symmetry, J. Math. Phys., 52 (2011), 032704.

[25] T. Takebe and L.P. Teo, Coupled modified KP hierarchy and its dispersionless limit, SIGMA, 2 (2006), 072.

[26] Y.Q. Yao, J.H. Zhang, R.L. Lin, X.J. Liu and Y, H. Huang, Bilinear identities and Hirota's bilinear forms for the $\left(\gamma_{n}, \sigma_{k}\right)$-KP hierarchy, J. Nonlin Math. Phys., 25 (2018), 309-323. 